\title{
Self-Organization at the Crack Tip of Fatigue-Resistant Thermoplastic Polyurethane Elastomers
}

\author{
Giorgia Scetta, Eric Euchler, Jianzhu Ju, Nathan Selles, Patrick Heuillet, Matteo Ciccotti,* \\ and Costantino Creton*
}

Cite This: Macromolecules 2021, 54, 8726-8737

Read Online

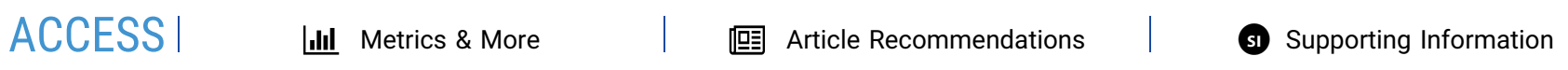

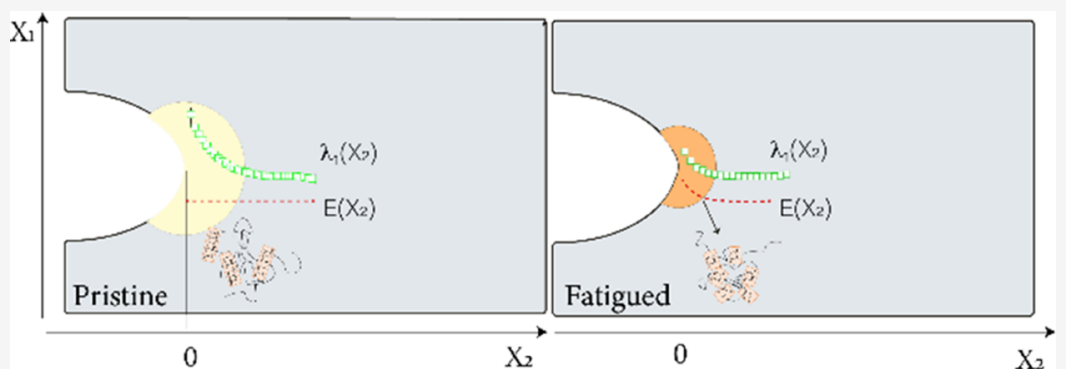

ABSTRACT: Despite their technological relevance, the resistance of soft thermoplastic polyurethanes (TPU) to crack propagation in cyclic fatigue has never been investigated in detail. In particular, a clear shortcoming in the literature for this class of materials is the lack of connection between the cyclic fatigue resistance and the large strain behavior that has a fundamental role in defining the material's resistance to crack propagation. We demonstrate here for the first time that when the strain-induced stiffening mechanism of TPU (already observed for large deformation) is combined with the presence of the nonhomogeneous strain, as in the case of cyclic fatigue, it produces a selective reinforcement in the crack tip area, which is the key to explain the remarkable cyclic fatigue resistance of TPU. Using commercial TPU with similar modulus $(\sim 8 \mathrm{MPa})$ but different large strain behavior, we show that the described mechanism stems from the multiphase nature of TPU and it is not necessarily linked to a specific large strain property as the case of TPU, which undergoes strain-induced crystallization.

\section{INTRODUCTION}

Thermoplastic polyurethanes (TPU) are segmented multiblock copolymers characterized by alternating blocks of soft segments (SS) and hard segments (HS). The former are composed of long and flexible polyester or polyether chains that ensure high deformability, while the latter consist of urethane-rich hard segments. The TPU typically self-organize in hard domains (HD), which are generated from the lateral stacking of HS through physical interactions and hydrogen bonding. They generally have dimensions of tens of nanometers, ${ }^{1,2}$ comparable to that of common reinforcing fillers used in rubbers. TPU can be produced in different grades with Young's moduli $E$ ranging from a few MPa to $1.000 \mathrm{MPa}$. Among those, soft TPU $(E<10 \mathrm{MPa})$, where the flexible chains represent the majority of the material and are physically cross-linked by the HD, generally show high elasticity and excellent abrasion resistance at ambient temperatures. Despite their high price, soft TPU have already found many applications in fields as sportswear and footwear and are gaining increasing industrial attention as recyclable alternative to replace classical filled and vulcanized rubber in structural applications such as cables, dampers, and belts. One of the most desirable properties in this kind of structural applications is the ability to sustain a large number of cyclic loadings at low stress levels without hazardous rupture of the material. In other words, they must have an appreciable cyclic fatigue resistance.

In a previous paper, ${ }^{3}$ we proposed a method to probe the cyclic fatigue resistance of soft TPU based on the classic fracture mechanics approach, originally developed for chemically cross-linked rubbers. We highlighted that during cyclic deformation, TPU experience a shake down ${ }^{4}$ and stabilization of the stress-stretch curve. Provided that the loading and unloading cycles are conveniently adapted to tackle the effects of plastic creep, the crack propagation process in standard notched pure-shear samples can be robustly expressed as crack propagation per cycle $\mathrm{d} c / \mathrm{d} n$ as a function of applied energy release rate $G$. These preliminary results confirmed the very high resistance of soft TPU to crack propagation under cyclic fatigue conditions even for large applied strains. To date, a

Received: April 28, 2021

Published: September 16, 2021

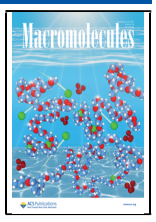


clear shortcoming in the literature for this class of materials comes from the lack of connection between the cyclic fatigue resistance and the large strain behavior that has a fundamental role in defining the material's resistance to crack propagation. Although the typical range of bulk strain used in fatigue experiments is generally considerably lower than the strain at break in uniaxial tests, the presence of the crack induces significantly larger local strains at the crack tip. ${ }^{5,6}$ This strain concentration is particularly important for TPU since their structure, and hence the mechanical response, evolves with applied strain. ${ }^{1,7-11}$ However, previous studies on the structural evolution of hard domains with applied strain only focused on uniaxial tension samples and did not include the effect of local singularities generated by defects or notches as is the case for fatigue fracture. Indeed, as proved by Mzabi et al. ${ }^{5}$ in filled and cross-linked styrene-butadiene rubber (SBR), the presence of a loaded crack generates a local strain gradient at the crack tip, the amplitude of which depends on external loading and on material's characteristics. To the best of our knowledge, a comprehensive characterization of the local morphology induced at the crack tip during a cyclic fatigue experiment, and the discussion of its effects on the crack propagation rate for soft TPU has never been carried out.

To understand the effect of a loaded crack on local structural modifications, it is useful to briefly recall some key results obtained in the uniaxial extension of TPU. ${ }^{7,9-16}$ Bonart $^{1}$ was among the first authors to investigate the deformation behavior of TPU using small- and wide-angle X-ray scattering, SAXS, and WAXS, respectively. He proposed that, for moderate levels of stretch $(\lambda<3)$, the progressive alignment of the SS along the tensile direction exerts a torque on the HD. As a result, the HD tend to orient in a transversal direction relative to the applied load. In a TPU with a low percentage of HS, further elongation generally corresponds to a reorganization of $\mathrm{HD}$ and alignment along the loading direction. This process, defined as "restructuring of the cross-linking" by Ishihara, ${ }^{12}$ consists of breaking and re-forming hydrogen bonds to realign the hard segments. An excellent summary on X-ray investigations in deformed TPU with low HS content (weight percentage about $12 \%$ ) was provided by Yeh and co-workers.

In this work, we tested the cyclic fatigue resistance of two TPU which share very similar small strain properties but present completely different large strain behavior in uniaxial tension at ambient temperature: TPU_XTAL which displays a marked strain-hardening partially due to strain-induced crystallization (SIC) and TPU_SOFT, which has higher extensibility and barely visible strain-hardening before rupture. In addition to the fatigue experiments, expressed by $\mathrm{d} c / \mathrm{d} n$ vs $G$ and uniaxial step-cycle tests, we used two additional techniques to characterize the differences between the bulk and at the crack tip as the sample is experiencing loading cycles in fatigue: digital image correlation (DIC) to characterize the strain field near the crack tip and spatially resolved in situ X-ray wide- and small-angle scattering analysis (WAXS, SAXS) to detect structural changes with the number of cycles both in the bulk and near the tip.

\section{MATERIALS AND METHODS}

Materials. The used TPU are commercial polyester-based polyurethane multiblock copolymers produced by BASF, Elastollan series, with the trade names: 565A 12P and LP9277 10, respectively, denoted as TPU_XTAL and TPU_SOFT based on their large strain behavior under uniaxial conditions. The materials were kindly provided by BASF.

Dog-bone samples had a cross section of $2 \mathrm{~mm} \times 4 \mathrm{~mm}$ and were cut along the injection direction from a $2 \mathrm{~mm}$ thick square-plate injection molded by the Laboratoire de Recherche et Contrôle des Caoutchoucs et Plastiques (LRCCP). The injection conditions are reported in Figure S1 and Table S1. The pure-shear geometry is generally used in cyclic fatigue experiments because the energy release rate $G$ can be easily calculated and is independent of crack length. ${ }^{17}$ All pure-shear samples were pre-notched using a fresh razor blade with a $20 \mathrm{~mm}$ cut. The chemical composition of both TPU is not available since they are commercial products. The number-average molecular weight $M_{\mathrm{n}}$ and weight-average molecular weight $M_{\mathrm{w}}$ of TPU_XTAL after injection are 47 and $61 \mathrm{~kg} / \mathrm{mol}$, respectively, and were obtained by gel permeation chromatography (GPC). The values of absolute molecular weights were extracted from refractive index and light scattering signals, using a measured $\mathrm{d} n / \mathrm{d} c$ value of $0.11 \mathrm{~mL}$. $\mathrm{g}^{-1}$ for the TPU. Fourier transform infrared (FTIR) spectroscopy was used for structural analysis of the polymers. Only in the case of TPU_SOFT, it revealed an absorbance peak around $1640 \mathrm{~cm}^{-1}$, which may be consistent with the presence of bidentate urea. The latter is generally associated with stronger interactions than simple hydrogen bonding in $\mathrm{HD}^{18}$ and may explain the poor solubility of TPU_SOFT suggesting a stronger interdomain stability.

Step-Strain Cyclic Tests. The dog-bone-shaped samples were strongly fixed between mechanical clamps since TPU are very tough. An optical detection system was used to measure the local stretch in the gauge area of the sample and to check the absence of slippage from the clamps during the test. The samples were loaded under uniaxial conditions at the stretch rate of $\dot{\lambda}=4 \mathrm{~s}^{-1}$. The loading was performed in a stepwise mode: 10 cycles were performed for each increasing value of maximum applied stretch $\lambda_{\mathrm{k}}$ for both TPU. The stress was reduced to $\sigma=0$ between two successive steps to prevent buckling. The mechanical quantities strain $\varepsilon$, stretch $\lambda$, Hencky strain $h$, nominal stress $\sigma$, and true stress $T$ are defined as below.

$$
\begin{aligned}
\varepsilon & =\frac{l-l_{0}}{l_{0}} \lambda=\frac{l}{l_{0}} h=\int \mathrm{d} \varepsilon=\int_{l}^{l_{0}} \frac{1}{l} \mathrm{~d} l=\ln (\lambda) \sigma=\frac{F}{A_{0}} T \\
& =\sigma \cdot(1+\varepsilon)
\end{aligned}
$$

Here, $l_{0}$ and $l$ indicate the initial length and instantaneous length, respectively; $A_{0}$ is the initial cross-sectional area; and $F$ is the measured force.

WAXS and SAXS Characterization. In situ WAXS and SAXS experiments were carried out under two conditions: on relaxed samples $(\lambda=1)$ and on strained samples $(\lambda=2.5)$. The first set of experiments $(\lambda=1)$ was carried out on a GANESHA 300XL+ system from JJ X-ray 1 in the X-ray lab at DSM Materials Science Center in the Netherlands. The in situ experiments on strained samples $(\lambda=$ 2.5) were carried out at the PETRA III beamline P03 at Deutsches Elektronen-Synchrotron (DESY) in Hamburg. The technical details of the scattering experiments are summarized in Table 1 .

The two-dimensional (2D) scattering data were integrated using the software tool FIT-2D. ${ }^{19}$ All data were corrected by subtracting background scattering. In situ WAXS and SAXS experiments were

\section{Table 1. Parameters of X-ray Experiments}

\begin{tabular}{lll}
\multicolumn{1}{r}{ facility } & DESY, Petra III & \multicolumn{1}{c}{ DSM resolve } \\
energy & $13 \mathrm{keV}$ & $8.4 \mathrm{keV}$ \\
wavelength $(\nu)$ & $0.096 \mathrm{~nm}$ & $0.15 \mathrm{~nm}$ \\
beam size & $22 \times 16 \mu \mathrm{m}^{2}$ & $300 \times 300 \mu \mathrm{m}^{2}$ (SAXS) \\
& & $700 \times 700 \mu \mathrm{m}^{2}$ (WAXD)
\end{tabular}

step width

$100 \mu \mathrm{m}$

distance from detector SAXS WAXS $4050 \mathrm{~mm} \quad 1465 \mathrm{~mm}$

image resolution 

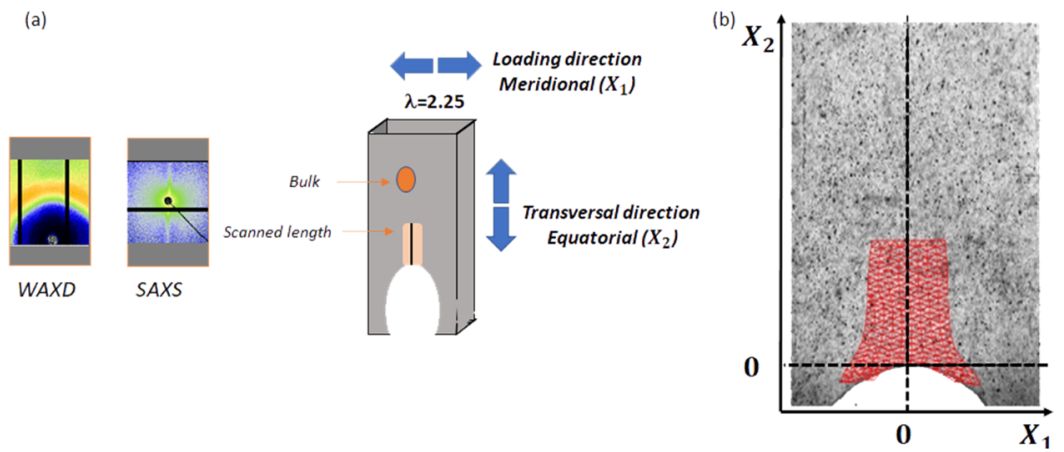

Figure 1. Sketch of the experimental setup for WAXD and SAXS in situ experiments (a). Example of open crack profile and deformed mesh obtained from DIC analysis (b). $X_{1}$ and $X_{2}$ indicate the directions parallel (meridional) and perpendicular (equatorial) to the applied load, respectively.

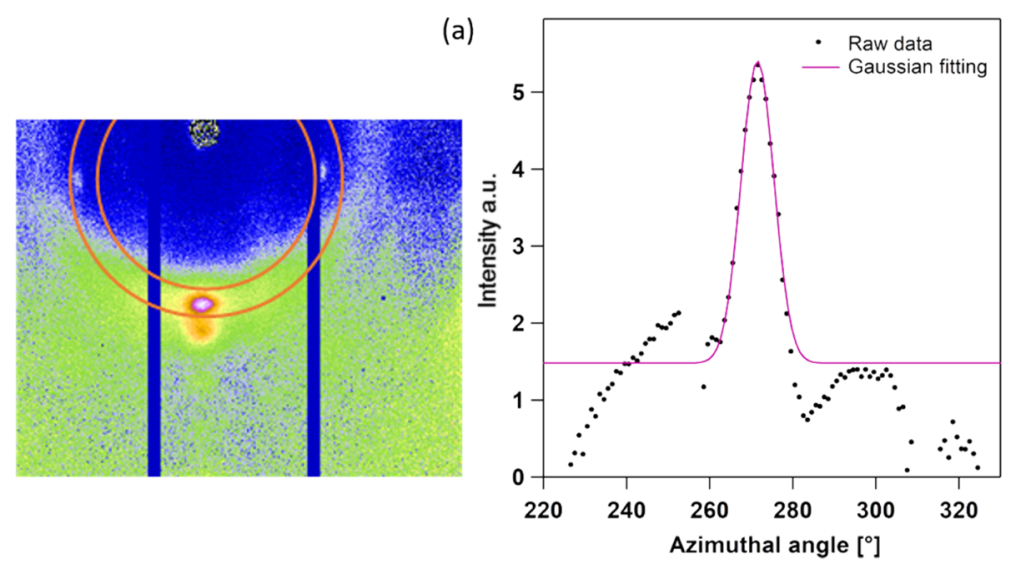

(b)

Figure 2. Example of azimuthal integration to evaluate the orientation factor (a). Example of Gaussian fitting of azimuthal one-dimensional (1D) profile to evaluate the FWHM (b).

carried out for two sets of samples: samples that were pre-fatigued and pristine samples. All of them contained a notch. For in situ experiments, the notched pure-shear specimens were stretched in a displacement-controlled mode up to $\lambda=2.25$, then 2D WAXS and SAXS scans were performed along the entire sample's length parallel to the crack and in front of it. The position of the first point (the actual crack tip) has an uncertainty of $\pm 100 \mu$ m, i.e., the step width between each successive scan. Figure 1a reports a schematic of the in situ scattering experimental setup. Figure $1 \mathrm{~b}$ is an example of the crack profile of the loaded sample and shows the deformed mesh used to evaluate the local strain at the crack tip. The axes $X_{1}$ and $X_{2}$ correspond to the direction parallel (meridional) and perpendicular (equatorial) to the applied load.

WAXS Analysis. The 2D WAXS pattern was circularly integrated. Peaks in the $2 \theta$ range between 12 and $20^{\circ}$ were deconvoluted using Gaussian/Lorentzian peak fitting routines. The crystalline fraction $\chi_{c}$ was evaluated classically as the ratio between the total area of crystalline peaks $I_{\mathrm{cr}}$ and the total area (crystalline and amorphous: $I_{\mathrm{cr}}$ $\left.+I_{\mathrm{am}}\right)$ underneath the diffraction profile as

$$
\chi_{\mathrm{c}}=\int_{2 \theta} \frac{I_{\mathrm{cr}}}{I_{\mathrm{cr}}+I_{\mathrm{am}}}
$$

Azimuthal integration was performed on the most prominent peak as indicated in Figure 2a. The obtained peak was then fitted with a Gaussian function to calculate the full width at half-maximum (FWHM) as in Figure 2b. The value of FWHM is indicative of the variability of the lattice parameter around its average value and it is an indication of the level of disorder of the structure. A high value of FWHM indicates less order in the crystal phase.

SAXS Analysis. SAXS data are expressed in terms of intensity as a function of wave vector $q=\frac{4 \pi \sin \theta}{\nu}$, where $2 \theta$ is the scattering angle and $\nu$ is the wavelength. The long period $L$ represents the recurrent spacing periodicity between hard domains, ${ }^{1}$ and it was calculated as $L=\frac{2 \pi}{q^{*}}$, where $q^{*}$ represents the center of the Gaussian fitting of the peak in the 1D SAXS profile.

Cyclic Fatigue Experiments. Fatigue results are generally expressed in terms of crack propagation per cycle $\mathrm{d} c / \mathrm{d} n$ vs $G$. All of the experiments were carried out on pure-shear samples following the procedure developed for TPU by Scetta et al. in a previous paper. The curve $G\left(\lambda_{\max }\right)$ was evaluated on un-notched pure-shear samples by applying sinusoidal cycles between $\sigma_{\min }=0$ and an increasing value of the maximum stretch $\lambda_{\max }$ at the frequency of $10 \mathrm{~Hz}$. Under cyclic conditions, the stress-stretch curve of TPU shows a very marked stress softening and some residual strain that eventually stabilizes between 5000 and 10000 cycles. This effect is known as shake down. ${ }^{4}$ We used the stress-stretch curve at 10000 cycles to evaluate $G\left(\lambda_{\max }\right)$ as $G=H W_{\mathrm{PS}}\left(\lambda_{\max }\right)$, where $H$ is the unstrained height of the pureshear sample and $W_{\mathrm{PS}}$ is the strain energy density as a function of the maximum applied stretch $\lambda_{\max }$ in the pure-shear geometry. ${ }^{17}$ To evaluate the crack propagation per cycle, a single long notch of $22 \mathrm{~mm}$ was cut on pristine samples. Each notched sample was strained between $\sigma_{\min }=0$ and $\lambda_{\max }$ for a minimum of 36000 cycles. During the test, the extension of the crack was monitored using a digital camera (BAUMER VCXU-32M) with a resolution of 3.1 Megapixel equipped with a macro-objective resulting in a pixel size of $38 \mu \mathrm{m}$. The crack propagation rate stabilizes after 5000-10000 cycles and then we evaluated $\mathrm{d} c / \mathrm{d} n$.

Digital Image Correlation (DIC). DIC is a technique that allows measuring displacement fields by matching a reference with a deformed image. Here, the Correli-LMT software, ${ }^{20}$ which represents the displacement field by the same kind of mesh as in finite element methods, was used. The final displacement is evaluated using an 

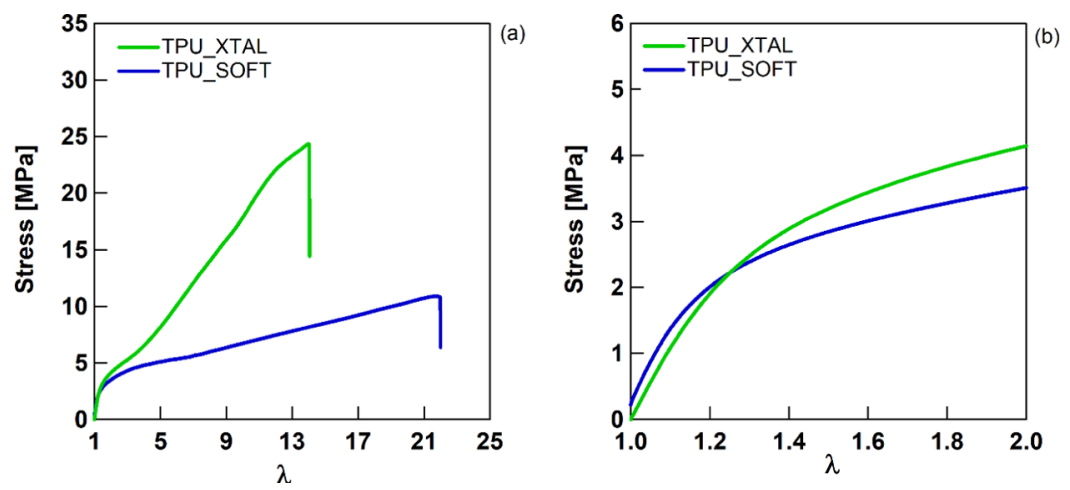

Figure 3. Uniaxial nominal stress-stretch curve of TPU_XTAL and TPU_SOFT at $23{ }^{\circ} \mathrm{C}$ (a). Zoom on the small stretch region (b).

algorithm that minimizes the difference in the gray levels between the matched images, while imposing some level of regularity to the solutions.

The objective adopted for this analysis corresponds to a pixel size of $7 \mu \mathrm{m}$ and the mesh size was $x$ chosen as 16 pixels. Under this condition, the spatial resolution of the DIC analysis (minimum distance between two adjacent estimates of displacement) was 112 $\mu \mathrm{m}$. Since the datapoint associated by convention with a distance of 0 $\mathrm{mm}$ from the crack tip is indeed the closest available point, this spatial resolution should also be considered as an uncertainty on the distance of such point from the crack tip. The samples were uniformly backlit. A random speckle texture was obtained by black ink spraying, a method which provides a good contrast with the sample surface (either white or transparent). For each DIC characterization, a set of 40-60 images was acquired between $\sigma_{\min }=0$ and $\lambda_{\max }$. To minimize the alteration of the gray levels by the large applied strains during DIC, the correlation procedure was first performed between each couple of subsequent images starting from the unloaded condition up to the maximum strain. Then, the total displacement for each $\lambda$ was evaluated by progressively adding the differential displacement fields of each step. The total displacement field was then used to evaluate the local strain field following the same procedure as in Mzabi et al. ${ }^{21}$

\section{RESULTS}

Tensile Tests. Figure 3a reports the stress-stretch curve in uniaxial tension at $23{ }^{\circ} \mathrm{C}$ and highlights the main differences in large strain behavior between TPU_XTAL and TPU_SOFT. Both TPU are characterized by a high extensibility at failure (more than 1000\%) and, after an initial linear elastic regime that only lasts a few percent strain, they soften as displayed in Figure $3 \mathrm{~b}$. In the case of TPU_XTAL, this is rapidly followed by a marked strain hardening, which is much less pronounced in TPU_SOFT. Table 2 reports the elastic moduli as well as the stress and stretch at break for both TPU.

Cyclic Fatigue. Figure $4 a$ shows the values of $d c / d n$ in the steady-state regime as a function of $G$ for both TPU. The threshold values, $G_{t}$, indicated in the picture, are conventionally evaluated using the same procedure adopted in our previous paper ${ }^{3}$ and correspond to the minimum value of $G$

Table 2. Tangent Modulus (E), Maximum Stretch $\left(\lambda_{\mathrm{b}}\right)$, and Maximum Nominal Stress $\left(\sigma_{\mathrm{b}}\right)$ at Break for TPU_XTAL and TPU_SOFT at $23{ }^{\circ} \mathrm{C}$

$\begin{array}{cccc}\text { name } & E[\mathrm{MPa}] & \lambda & \sigma_{\mathrm{b}}[\mathrm{MPa}] \\ \text { TPU_XTAL } & 8.7 & 15.4 & 25.1 \\ & \pm 0.1 & \pm 1.2 & \pm 1.3 \\ \text { TPU_SOFT } & 7.8 & 19.1 & 11.5 \\ & \pm 0.1 & \pm 2.8 & \pm 0.8\end{array}$

below which during a single fatigue experiment lasting 36000 cycles and with the resolution of our optical system $(38 \mu \mathrm{m})$, we could not detect any crack propagation. Under this condition, the minimum detectable crack growth per cycle was equal to: $\frac{38 \mathrm{~m}}{36000 \text { cycles }} \sim 1 \mathrm{~nm} /$ cycle. Using this definition, the values of $G_{\mathrm{t}}$ were found to be $\sim 3$ and $\sim 5 \mathrm{~kJ} / \mathrm{m}^{2}$ for TPU_SOFT and TPU_XTAL, respectively. We underline that this is not a physical value of $G_{t}$, but it depends on the experimental system, and we cannot exclude that higher resolution and/or longer testing may lead to a lower value of $G_{\mathrm{t}}$. Nevertheless, in classical rubbers, the average threshold $G_{\mathrm{t}}$ is typically found between 40 and $100 \mathrm{~J} / \mathrm{m}^{2,22,23}$ well below those obtained for TPU, which present a fatigue threshold (and the corresponding stretch $\lambda$ ) more than 1 order of magnitude larger. Above the threshold, $\mathrm{d} c / \mathrm{d} n$ of TPU_XTAL increases as a power law $\approx G^{2.7}$. This behavior is similar to that found in several elastomeric systems, where the exponent $n$ varies between 1 and $4 .{ }^{24}$ On the other hand, TPU_SOFT has a rapid transition between a very slow- or almost not propagating regime to a fast-propagating regime, where the values of $\mathrm{d} c / \mathrm{d} n$ are only weakly dependent on $G$ and are higher than that for TPU_XTAL at comparable G. The toughness, generally defined as a critical value $G_{c}$ or $\Gamma$ above which the crack propagates so fast that the sample breaks in a few cycles, ${ }^{25}$ must be above $20 \mathrm{~kJ} / \mathrm{m}^{2}$ in both TPU since this unstable regime was not observed. For comparison, for a typical filled natural rubber with similar linear modulus, the average value of toughness is generally below $10 \mathrm{~kJ} / \mathrm{m}^{2}{ }^{25}$ Overall, both TPU have a higher fatigue resistance than classical filled elastomers at the same applied $G$ and require a much higher strain to propagate the crack during cyclic fatigue. Figure $4 \mathrm{~b}$ reports an equivalent representation in terms of $\mathrm{dc} /$ $\mathrm{d} n$ vs the maximum stretch $\lambda_{\max }$ which demonstrates the extremely large strain required to propagate a crack in cyclic fatigue for TPU. Interestingly, comparable values of $\mathrm{d} c / \mathrm{d} n$ for a similarly high value of $\lambda_{\max }$ were reported by $\mathrm{Li}$ et al. ${ }^{26}$ in a hydrogel material, which, similarly to TPU, presents a multiphase morphology. They showed that highly fatigue resistance hydrogels containing a reinforcing hard phase at the $100 \mathrm{~nm}$ scale, much larger than the characteristic size of the polymer network defined as the characteristic distance between cross-links $(10 \mathrm{~nm})$, can be deformed at $\lambda_{\max }>1$ for thousands of cycles without initiating a catastrophic propagation of the crack. In those systems, the maximum stretch is a critical parameter in determining the resistance of the material against fatigue. 

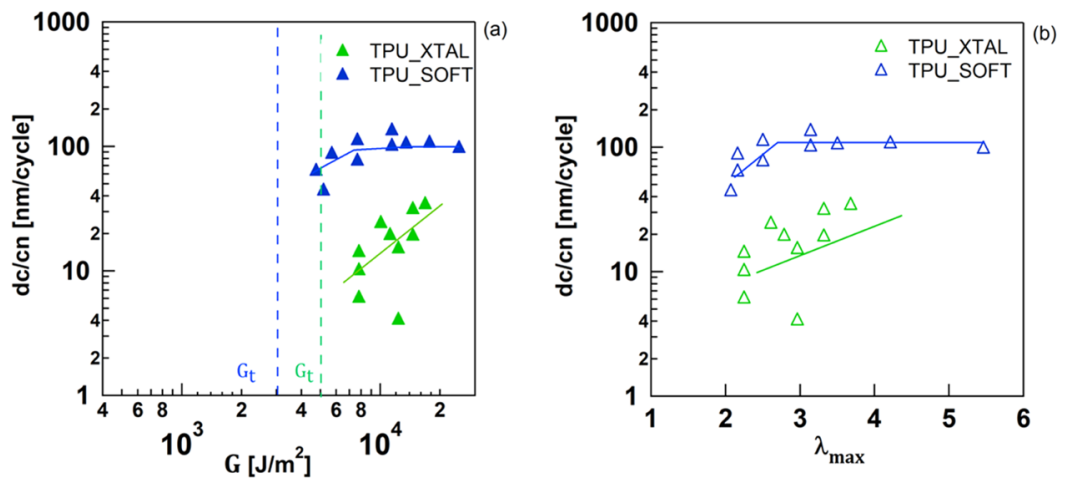

Figure 4. Crack growth rate per cycle as a function of the applied release rate $G(a)$ and applied stretch $\lambda_{\max }$ (b) for TPU_XTAL and TPU_SOFT at $23{ }^{\circ} \mathrm{C}$.

Stiffening Effect in Cycles of Uniaxial Deformation. Characterizing the large strain cyclic behavior is fundamental to better understand the fatigue resistance of TPU. In cyclic fatigue, the crack propagates at levels of bulk applied stretch considerably lower than the strain at break in quasi-static extension. Despite that, close to the crack tip, the local strain is higher than in the bulk because of the stress and strain concentration induced by the crack. The history of a material point being approached by the propagating crack during cyclic fatigue can be simplistically represented by step-strain cyclic experiments up to rupture. In a previous paper, ${ }^{27}$ we performed cyclic uniaxial loading at increasing values of maximum stretch for the same TPU used in this work. We demonstrated that the dissipative behavior in the uniaxial extension of TPU_SOFT and TPU_XTAL is qualitatively very similar and cannot thus explain in any obvious way the considerably higher fatigue resistance of TPU XTAL. As explained in our previous work, ${ }^{27}$ during the cyclic experiments, the stress-strain response of the material is permanently affected by the maximum applied strain and it does not follow the classical trend observed in cross-linked elastomers. We confirmed this result with two different indicators both at large and small strain, and in Figure 5, we

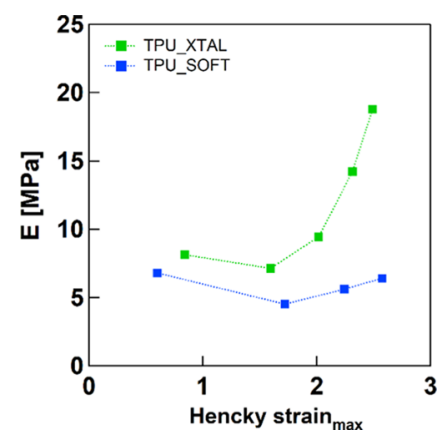

Figure 5. Tangent modulus $E$ vs maximum Hencky strain for both TPU at $23{ }^{\circ} \mathrm{C}$.

report the evolution of the tangent modulus $E$ (calculated at low strain) with $\lambda_{c}$ as often done to estimate damage in elastomers. For both TPU, E first decreases, as commonly observed in filled elastomers, ${ }^{28}$ and above a critical strain, it increases again achieving the same or higher value than the pristine sample. Overall, these results indicate a permanent stiffening effect, which combined with a residual deformation strongly suggests a strain-induced modification in the TPU microstructure. Figure 5 also shows that the stiffening effect in TPU_XTAL, which can crystallize under strain, is considerably more pronounced than in TPU_SOFT for all values of applied maximum stretch. To understand the role of the increase of the elastic modulus with applied strain in TPU under cyclic fatigue, we carried out a complete evaluation of the strain field and of the reorganization of the microstructure at the crack tip before and after fatigue cycling in TPU_XTAL and TPU_SOFT.

Local Strain Gradient in TPU before and after Fatigue. Figure 6 reports the value of the local stretch $\lambda_{1}$ in the loading direction calculated at different distances from the crack tip for both TPU by DIC. Two sets of samples were used: pristine and fatigued. Pristine samples were directly strained at $\lambda_{\max }=2.25$, while fatigued samples were previously cycled for 36000 cycles and then monotonically strained at the same value of $\lambda_{\max }$.

For comparable distances from the crack tip, the value of vertical strain $\lambda_{1}$ of the fatigued sample is lower than that of its pristine counterpart in both TPU. However, in the case of TPU_XTAL, the strain localization is much less pronounced after fatigue and only involves a smaller area around the crack $(\approx 1000 \mu \mathrm{m})$, compared to TPU_SOFT $(>2000 \mu \mathrm{m})$. This result is consistent with the more pronounced cyclic strainstiffening effect of TPU_XTAL reported in Figure 5. At the beginning of the test, the action of repeated cycles at the same value of $\lambda_{\max }$ gradually modifies the local response of the material, which eventually becomes stiffer and less stretchable at the crack tip explaining the lower values of $\lambda_{1}$ in fatigued samples. This effect is also accompanied by an overall induced uniformization of the strain gradient after fatigue as shown in Figure 7 , which reports the strain distribution of $\lambda_{1}$ along the direction $X_{1}$, parallel to the applied load, in the close vicinity of the crack tip. An additional remark must be made concerning the value of $\lambda_{\max }$ in fatigued samples. As shown in our previous work, ${ }^{3,27}$ TPU generally present some residual strain when cyclically loaded in uniaxial tension. The latter implies that the actual length $l_{0}^{\text {fatigued }}$ of unloaded samples after fatigue is longer than the original in pristine sample $l_{0}$, which we used to evaluate $\lambda_{\max }$. Therefore, the real stretch experienced by the material is lower than $\lambda_{\max }$. This effect is visible in Figure 6 for both TPU, where, for large distances from the crack tip, the value of $\lambda_{1}$ at the plateau (which corresponds to the macroscopic applied $\left.\lambda_{\text {max }}\right)$ is slightly higher in pristine than in fatigued sample. This effect is however barely distinguishable and cannot explain the remarkable reduction of the strain singularity after fatigue. 

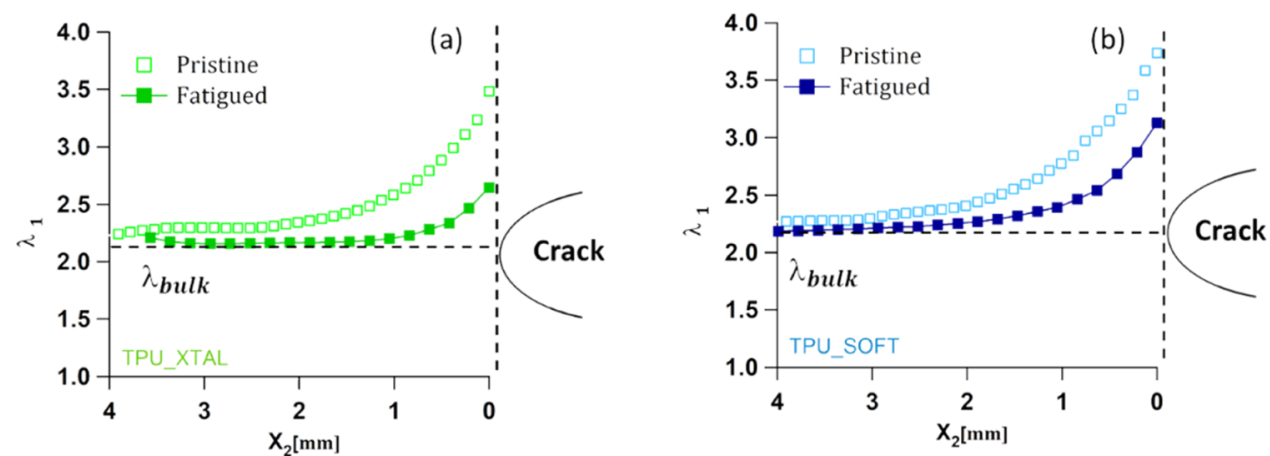

Figure 6. Maximum stretch in the loading direction $\lambda_{1}$ as a function of the distance from the open crack tip evaluated for TPU_XTAL (a) and TPU_SOFT (b), both pristine and after being fatigued for 36000 cycles at $\lambda_{\max }=2.25$.

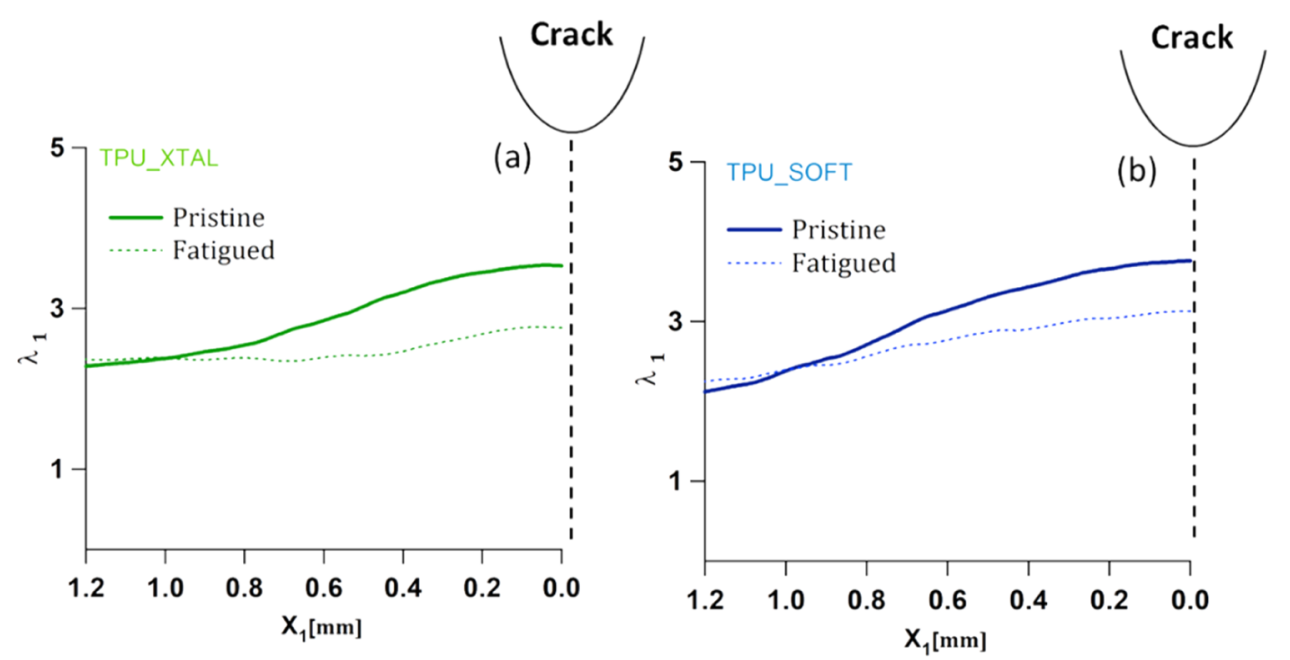

Figure 7. Distribution of the maximum $\lambda_{1}$ at the crack tip along the loading direction $X_{1}$ for pristine and fatigued TPU_XTAL (a) and TPU_SOFT (b).

Effect of the Strain Gradient on the Microstructure in Pristine and Fatigued TPU. As previously explained, the mechanical properties of TPU are intrinsically related to the hard-soft domains organization. The natural physical explanation for the overall reduction in the intensity of the strain singularity in the crack tip zone in fatigued TPU must be found in the peculiar microstructural rearrangements induced by cyclic loading.

In situ WAXS and SAXS experiments were carried out at different distances from the crack tip on notched pure-shear samples of TPU_XTAL and TPU_SOFT loaded at $\lambda_{\max }=2.25$ as detailed in the Materials and Methods section. Also, in this structural analysis, two sets of samples were used: pristine and fatigued as already described for the analysis by DIC. We first discuss notched pristine samples loaded to $\lambda_{\max }=2.25$. Figure 8 a shows representative WAXS and SAXS 2D patterns at two different sample locations, far away (bulk) and very close to the crack tip, obtained for pristine TPU_XTAL (the corresponding images for TPU_SOFT are reported in Figure S2). A first qualitative analysis of the $2 \mathrm{D}$ patterns reveals two important phenomena: (i) the development of a strain-induced anisotropy both in the bulk and in the crack tip zone and (ii) the change in the structure of the material as the local strain gradient develops. More specifically:

(i) The anisotropic character of strained TPU_XTAL is visible in all diffraction patterns. The darker area along the meridional direction in the circular 2D WAXS images indicates that the amorphous SS gradually orient toward the loading direction. ${ }^{7}$ Additionally, the presence of two meridional sharp reflections (or lobes) in the SAXS images indicates the preferential tilting of the HD along the meridional (loading direction) axis. $7,10,29$

(ii) The dissimilar morphology developed as the strain gradient increases is demonstrated by the different patterns observed in the bulk and in the crack tip region. In the 2D SAXS patterns corresponding to the crack tip area, the two meridional lobes are clearly less intense, while a new equatorial sharp streak appears. The appearance of the streak can be associated with either the presence of voids ${ }^{30,31}$ or a fibrillar morphology ${ }^{32}$ and is frequently found in TPU deformed in large strains. $^{7-10,14,29}$ This feature stems from the destruction of HD in favor of rod-like soft domains composed of both elongated SS and HS in the most strained region at the crack tip. A similar behavior was also observed in TPU_SOFT (Figure S2). The 1D WAXS integrated profiles for TPU XTAL and TPU SOFT are reported in Figure $8 b, c$. In the case of TPU XTAL (Figure $8 b$ ), the 1D WAXS profile close to the crack tip shows the presence of two main crystalline peaks, absent in the bulk, indicating the presence of well-oriented straininduced crystallization (SIC $)^{29,33}$ in the higher stretched area (see DIC data in Figure 6). On the other hand, crystalline peaks are clearly detected in the bulk and still 
(a)
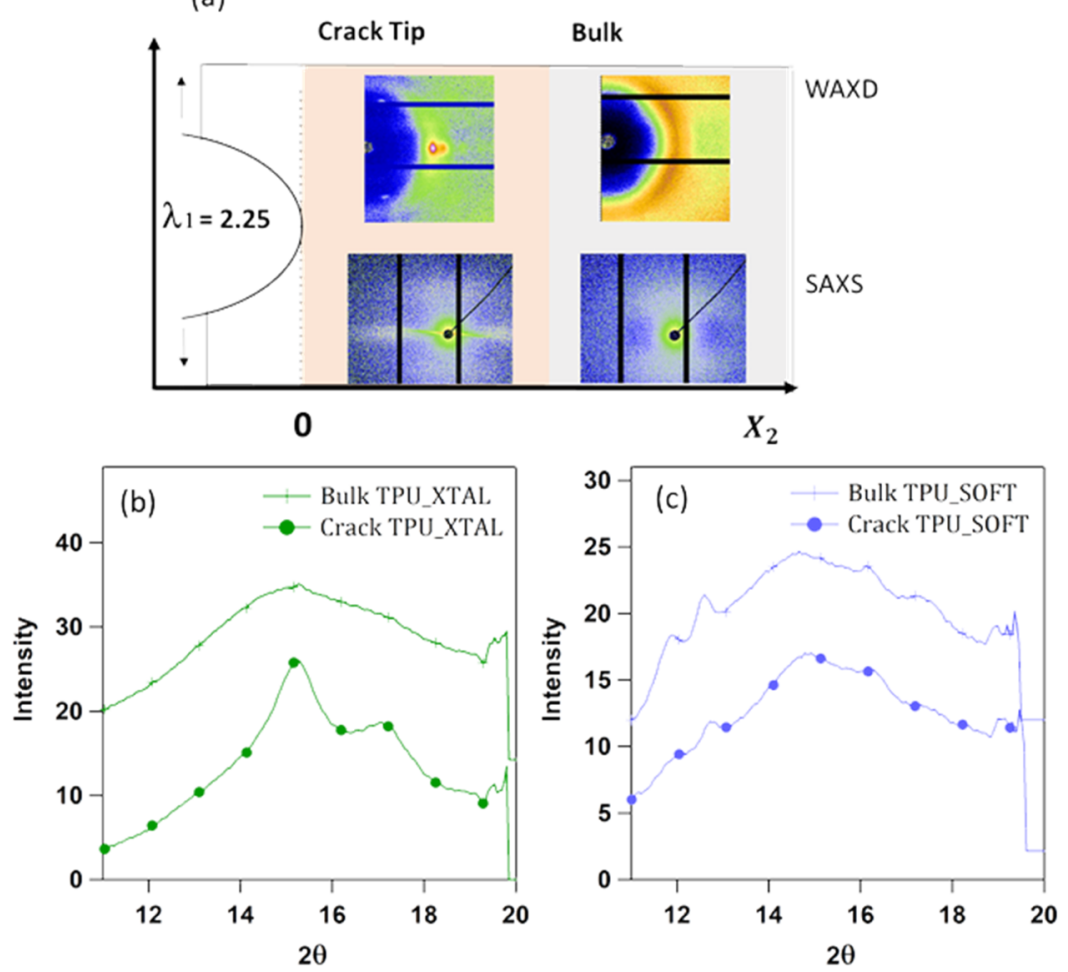

Figure 8. Representative in situ WAXD and SAXS 2D pattern for TPU_XTAL close and far from the open crack for pristine sample monotonically strained at $\lambda_{\max }=2.25$ (a). Integrated 1D profile of WAXS crystalline peak in TPU_XTAL (b) and TPU_SOFT (c) obtained for the crack tip and the bulk area at $\lambda_{\max }=2.25$.
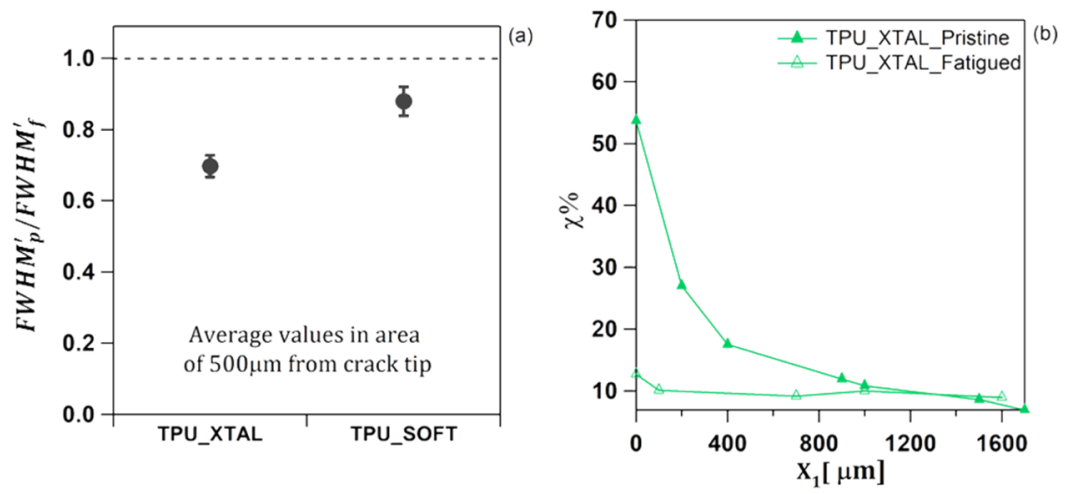

Figure 9. Values of averaged FWHM ratio between pristine and fatigued sample (a) and crystalline fraction in TPU_XTAL at increasing distance from the crack for pristine and fatigued sample (b) monotonically strained at $\lambda_{\max }=2.25$.

visible close to the crack tip for TPU_SOFT in the 1D WAXS profile of Figure 8c. The position of these peaks corresponds to those of the crystallized butadiene terephthalate $^{29}$ (PBT) sequence probably present as a comonomer in TPU_SOFT. This is however a hard segment (crystalline in the absence of strain) becoming less crystalline with strain.

To evaluate how the effect of several cycles affects the local structure evolution in TPU, we now compare the X-ray scattering results obtained at a similar distance from the crack tip for pristine and fatigued samples of TPU strained at $\lambda_{\max } \approx$ 2.25. Consistent with the DIC results of Figure 6, we find that the level of orientation and crystallinity is lower for fatigued samples than for pristine ones. Figure 9a shows in particular the ratio between peak widths $\mathrm{FWHM}_{\mathrm{p}}^{\prime}$ and $\mathrm{FWHM}_{\mathrm{f}}^{\prime}$, where FWHM $^{\prime}$ indicates the averaged FWHM values of the peak integrated along the azimuthal direction (as indicated in the Materials and Methods section) in the region within $500 \mu \mathrm{m}$ of the crack tip and the subscripts $\mathrm{p}$ and $\mathrm{f}$ stand for the pristine and fatigued sample, respectively. Values lower than unity indicate that the quality of the crystal orientation in the pristine sample is higher than that for the fatigued sample, at a comparable distance from the crack tip. This implies that the crystallites that were either produced during strain (TPU_XTAL) or originally present in the hard domains (TPU_SOFT) become fragmented and less oriented during fatigue. In the case of TPU_XTAL, we can also extract the crystalline fraction $\chi$, at different distances from the crack tip for both pristine and fatigued samples (Figure 9b). It is striking to see that while strain-induced crystallization is very active in pristine samples ( $\chi$ gradually increases when approaching the crack tip), the degree of crystallinity at the crack tip almost completely 

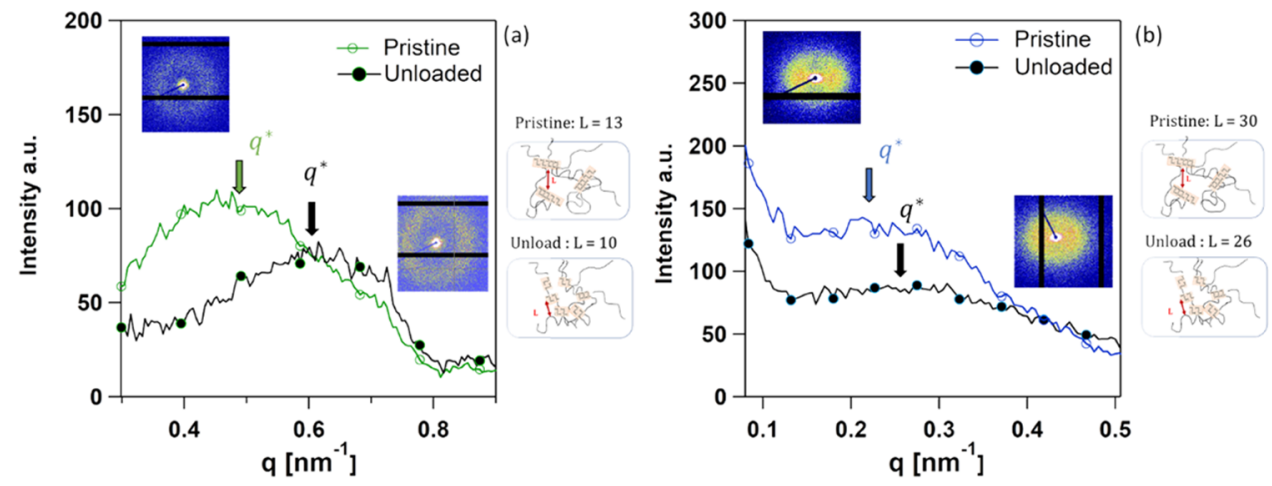

Figure 10. Two-dimensional SAXS images and the corresponding 1D profile for pristine and unloaded fatigued (after 36000 cycles) TPU_XTAL (a) and TPU_SOFT (b). The schematics show the decrease of $L$ and the fragmentation of the HD structure before and after fatigue testing (not all soft segments are represented for clarity). The X-ray patterns were collected at DSM, the Netherlands.

disappears in fatigued samples (Figure 9b). After being fatigued, both TPU_SOFT and TPU_XTAL are less oriented and, in the case of TPU_XTAL, show less strain-induced crystallinity when stretched again. This reduced ability of the cycled sample to orient and crystallize under stretch is somewhat counterintuitive, considering the remarkable fatigue resistance showed by both TPU, and especially by TPU_XTAL. Since the fraction of strain-induced crystallites increases with the applied strain, ${ }^{34}$ we believe that the lower crystalline fraction found in fatigued sample is a direct consequence of the reduced strain concentration (and therefore a lower value of maximum strain) generated at the crack tip during cyclic fatigue.

Permanent Modification of the Microstructure Induced by Strain History. In the previous section, we showed that loading cycles induce some reorganization of the original two-phase structure of TPU when stretched at the same value of $\lambda_{\max }=2.25$. We now investigate whether the original morphology is completely recovered when the sample is unloaded. To understand which changes are permanent after the removal of the external load, we compare the scattering patterns of TPU in its relaxed state $(\lambda=1)$ for pristine and fatigued sample with those obtained at $\lambda_{\max }=2.25$. Figure 10 shows 2D SAXS images and the corresponding 1D scattering profiles for TPU_XTAL (a) and TPU_SOFT (b). In both cases, the $1 \mathrm{D}$ scattering profile is shifted at higher $q$ for the unloaded fatigued sample; therefore, the interdomain distance $L$ related to the periodicity of the hard and soft phases reduces slightly for unloaded fatigued sample. The decrease of $L$ can be interpreted as permanent fragmentation of $\mathrm{HD}$ in smaller units during the fatigue experiment ${ }^{10}$ as shown in Figure 10a,b for TPU XTAL and TPU_SOFT, respectively. It is worthy to remark that the SAXS analysis for the unloaded sample was performed around 30 days after the sample was fatigued. This led us to believe that such fragmentation is not easy to recover at ambient temperature even for materials that present some relaxation effects as physical cross-linked TPU.

The fragmentation of HD is not the only permanent effect induced by cyclic loading on TPU. In the case of TPU_XTAL, the new crystalline phase formed near the crack tip during cycling is partially retained after fatigue as shown by the differential scanning calorimetry (DSC) thermograms comparing pristine TPU_XTAL and a pure-shear sample that was previously fatigued (Figure 11). As also reported by other authors, ${ }^{35}$ the endothermic peak $\left(\mathrm{T}_{\mathrm{m} \_ \text {SIC }}\right)$ absent in the pristine sample is associated with a new crystalline phase

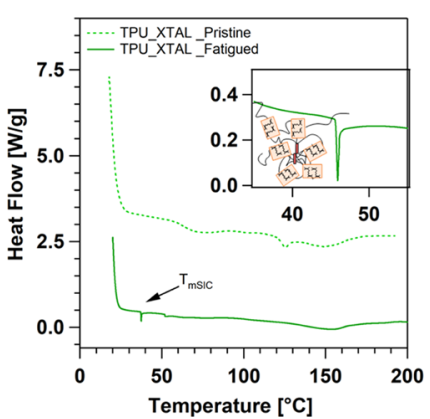

Figure 11. DSC thermogram for TPU_XTAL: pristine and fatigued sample. The inset is a magnification of the endothermic peak in the fatigued sample corresponding to the melt of crystallites schematically represented as red bars.

generated during loading, which persists after the removal of the load (red bars in the schematic of Figure 11).

\section{DISCUSSION}

Model To Explain the Strain-Induced Reinforcement in TPU. In strained TPU containing an initial crack, the strain singularity along the crack direction $X_{2}$, expressed as the stretch field $\lambda_{1}\left(X_{2}\right)$ evaluated at different distances $X_{2}$ ahead of the crack tip, is accompanied by a progressive change in the microstructure as proved by X-ray analysis. The changes in local microstructure with applied uniaxial strain in TPU have been investigated in-depth in the past for homogeneously strained samples. $7,10,11,13,14,29,33,36$ Different authors showed that some of those changes are permanent and that the unloaded material does not completely recover its original state, $^{10,16,29}$ but, depending on the maximum experienced strain, the polymer retains a certain degree of anisotropy or strain-induced crystallites as shown by the DSC analysis in Figure 11. In particular, for TPU made from SS able to crystallize under strain, the formation of new crystalline domains, which are persistent after unloading, may be responsible for the strengthening of the material with increasing strain. In our case, the modulus of both TPU increases with applied strain, but X-ray analysis shows that only TPU XTAL crystallizes under strain. This indicates that the strengthening effect induced by the strain is not uniquely related to the formation of a crystalline phase, but is a general property due to the self-organized structure of TPU in soft and hard domains. We believe that the permanent reorganization of the two-phase microstructure in TPU induces stiffening of the 


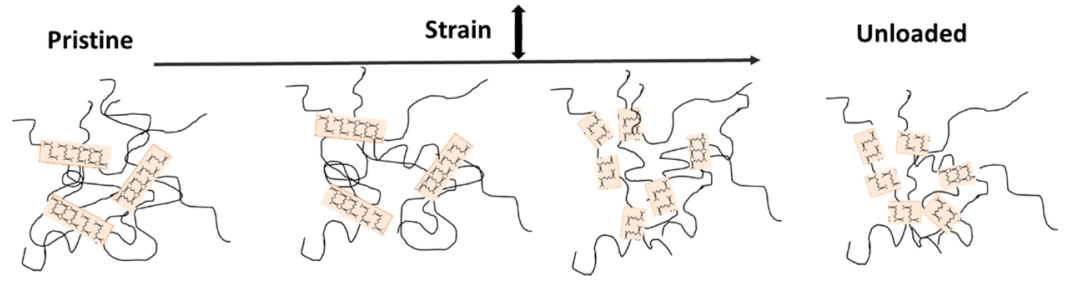

Figure 12. Sketch of the effect of elongation on HD restructuration.

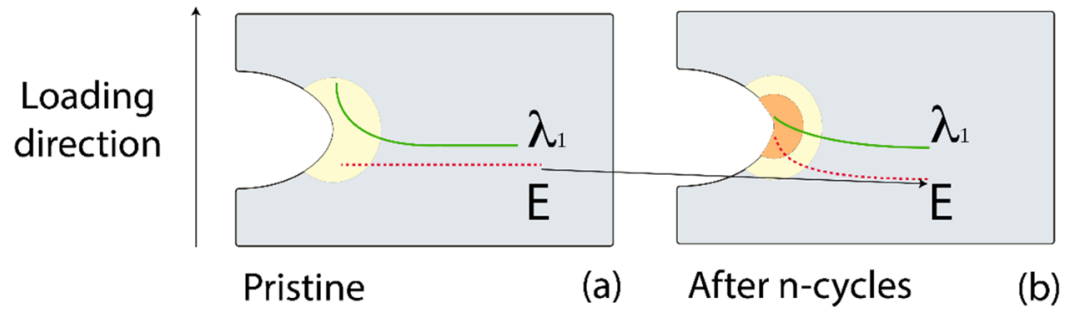

Figure 13. Sketch of the spatial evolution of the tangent modulus $E$ and strain $\lambda_{1}$ for pristine and fatigued TPU. In a notched and loaded pristine TPU, $E$ is constant and a strain gradient is induced by the presence of the crack in the neighboring area (a). After thousands of cycles, the reorganization at the crack tip generates a hard core with a higher local modulus than the bulk, which strengthens the crack tip reducing the strain field compared to the loaded pristine sample (b).

material through two main possible mechanisms, which in some cases can act simultaneously:

(1) Above a certain value of $\lambda_{\max }$ the original HD are fragmented into smaller units and this new structure partially persists after the removal of the strain (as suggested by the decrease of the long period for strained TPU (Figure 10)). The fragmentation of HD may contribute to the increase in stiffness in two different and complementary ways:

(A) Filler-like effect of HD. In a homogeneously stretched sample at the same "filler volume fraction", these smaller and probably well-dispersed HD contribute more to the modulus in analogy with a nanoparticle effect.

(B) Physical cross-linking effect of HD. In classical crosslinked elastomers, the elastic modulus is defined by the number of elastically active chains per unit volume. In pristine TPU, we can consider that not all of the polymer chains are elastically active and some of them are coiled in closed loops. When HD are fragmented in smaller units, some elastic chains can be released from their original conformation as schematically shown in Figure 12. In this case, although the density of elastic soft segment chains remains unchanged, the number of elastically active ones can increase causing an increase of the modulus. The reloaded sample thus shows both an increase in tangent modulus and a decrease in the stretch at break with applied strain as shown in our previous paper. ${ }^{27}$

(2) In this new reconfiguration of $\mathrm{HD}$, some soft chains, which were highly elongated during the deformation of the sample, may be prevented from recovering their original configuration, thus remaining partially elongated in the unloaded sample explaining the presence of residual crystallinity in TPU_XTAL. This residual crystalline phase acts as additional hard domains as suggested by the larger increase of $E$ (and reduction of $\left.\lambda_{1}\right)$ in TPU_XTAL. This leads to a further increase of the fatigue resistance compared to noncrystallizing TPU, but it is not a necessary condition for strain-induced reinforcement of TPU.

Effects Related to the Presence of a Crack in Cyclic Deformation. Previous investigations on the structural evolution of soft TPU with applied strain mainly focused on homogeneously strained materials and sometimes discussed a remarkable strain-stiffening after elongation. ${ }^{10}$ To the best of our knowledge, the effect of the presence of a crack and its associated strain localization has never been investigated in this context. In Figure 6, we have shown that the presence of a strain gradient at the crack tip causes a corresponding spatial gradient in the structural reorganization within the TPU, thus affecting the local mechanical response to the applied strain. Intriguingly, we also observed that the repetition of several loading cycles (at the same bulk $\lambda_{\max }$ ) leads to a decrease of the strain-induced anisotropy and crystallinity fraction (in TPU_XTAL) in the crack tip region and, in general, to a decrease in the sharpness of the structural gradient. We infer that the less oriented structure created by cyclic fatigue at the crack tip, compared to their pristine counterpart, is associated with a reduction of the severity of the strain concentration at the crack tip with number of cycles. Obviously, in cyclic fatigue, the strain experienced in the bulk is lower than that close to the crack tip, which in turn controls the crack propagation rate. This difference in strain generates a spatially heterogeneous structure consisting of the region far from the crack, which is deformed at below $\lambda_{\mathrm{c}}$ (and similarly to classical elastomers has tangent modulus $E$ lower than the pristine material, see Figure 5) and a stiffer core in the crack tip region, where the strain eventually may become large enough to overcome the critical $\lambda_{\mathrm{c}}$ generating an increase in $E$, which locally becomes equal to (TPU_SOFT) or higher (TPU_XTAL) than the pristine one. This difference in stiffness produced by the spatial-dependent restructuration of hard domains can be consequently interpreted as a sort of protecting mechanism, which, after a short transitory, shields the crack tip from achieving a very large strain, which may produce the rupture of material and therefore is correlated to crack propagation. Because of the stiffened core, the material 
far from the crack in the fatigued TPU must deform more to reach the same local strain around the crack tip compared to the pristine sample and results in a decrease in the severity of the strain concentration at the crack tip as visible in Figures 6 and 7. The proposed scenario is schematically shown in Figure 13 and is consistent with the lower crystallinity observed in TPU_XTAL after fatigue.

Noteworthy, the equatorial streak that we observed in the SAXS 2D pattern for the area close to the crack tip (Figure 8) above a characteristic stretch $\left(\lambda_{\max }>2.25\right)$ is characteristically seen by X-ray scattering in several $\operatorname{TPU}^{1,7,8,10}$ and was associated with the presence of either fibrillar structures or voids, which could be seen as a signature of certain damage produced in the system. For moderate applied macroscopic strains, this streak is only visible in the crack tip regime. However, when the whole sample is strained above this stretch threshold, some structural damage can take place in the bulk causing a ruin of the sample.

Effect of Crack Tip Stiffening on the Energy Available for Crack Propagation in Fatigue. We should finally discuss how the local spatial reorganization is related to the energy available for crack advance in cyclic fatigue. Mzabi et al. ${ }^{5}$ proposed the concept of local energy release rate $g_{\text {local }}$ indicating the locally stored elastic energy available for crack propagation in different SBR. They considered the highly strained zone at the crack tip as homogeneously strained at $\varepsilon_{\max 11}$ (corresponding to the maximum strain detected by DIC). $g_{\text {local }}$ was estimated as follows

$$
g_{\text {local }}=W\left(\varepsilon_{\max }\right) h_{0}
$$

where $\varepsilon_{\max }$ is the maximum strain calculated with DIC at the crack tip, $W\left(\varepsilon_{\text {max }}\right)$ indicates the elastic strain energy $W\left(\varepsilon_{\max }\right)=\int_{0}^{\varepsilon_{\max }} \alpha \mathrm{d} \varepsilon$ obtained from the uniaxial tension curve, and $h_{0}$ is the size of the strain concentration region in the loading direction. In that paper, they show that different SBR, differing by almost 2 orders of magnitude in terms of $\mathrm{dc} /$ $\mathrm{d} n$ vs $G$, fall on a master curve when $\mathrm{d} c / \mathrm{d} n$ is plotted against $g_{\text {local }}$. In particular, the highest fatigue-resistant SBR presents both a lower level of local strain at the crack tip and less elastic energy available at the crack tip ( $\left.g_{\text {local }}\right)$ for the same value of applied $G$. We decided to apply the proposed method $^{3}$ to calculate the local energy available at the crack tip $g_{\text {local }}$ in the pristine and fatigued TPU loaded at $\lambda_{\max }=2.25$. According to Mzabi et al., ${ }^{5} h_{\mathrm{o}}$ corresponds to the portion of the material in the loading direction where $\varepsilon_{\max }>0.95 \varepsilon_{11}$ and was calculated from the data of Figure 7. We used the 1st and 10th cycles to evaluate $W\left(\varepsilon_{\max }\right)$ (and therefore $\left.g_{\text {local }}\right)$ in pristine and fatigued samples, respectively. The choice of using the 10th cycle of the sample under uniaxial loading to evaluate $g_{\text {local }}$ for the fatigued sample is justified by the fact that the major changes in the cyclic stress-strain curve of the TPU take place at the beginning of the test. All of the values are reported in Table 3. Noteworthy, in the case of the fatigued sample, $g_{\text {local }}$ provides only an upper bound value of the strain energy density experienced by the material at the crack tip. Indeed, as discussed in our previous paper, during cyclic loading, the strain energy $G$ reduces with number of cycles. The most dramatic drop in $G$ vs cycle occurs within the first cycles and then gradually achieves a steady state after some thousands of cycles. For the same maximum applied strain, pristine TPU_SOFT, which has a lower fatigue resistance than TPU_XTAL, has higher values of $g_{\text {local }}$. Moreover, the
Table 3. $\varepsilon_{\max 11}, h_{\mathrm{o}}, W\left(\varepsilon_{\max }\right)$, and $g_{\text {local }}$ for TPU_SOFT and TPU_XTAL for Pristine (P) and Fatigued (F) Sample Strained at $\lambda_{\text {max }}=2.25$ Corresponding to Steady State $G$ of 5600 and $7800\left[\mathrm{~J} / \mathrm{m}^{2}\right]$, Respectively

\begin{tabular}{lllccc} 
& $\begin{array}{c}G \\
{\left[\mathrm{~J} / \mathrm{m}^{2}\right]}\end{array}$ & $\varepsilon_{\max 11}$ & $\begin{array}{c}h_{\mathrm{o}} \\
{[\mathrm{mm}]}\end{array}$ & $\begin{array}{c}W\left(\varepsilon_{\max }\right) \\
{\left[\mathrm{MJ} / \mathrm{m}^{3}\right]}\end{array}$ & $\begin{array}{r}g_{\text {local }} \\
{\left[\mathrm{J} / \mathrm{m}^{2}\right]}\end{array}$ \\
TPU_SOFT_P & & 2.8 & 0.58 & 3.69 & 2143 \\
TPU_SOFT_F & 5600 & 2.13 & 0.78 & 1.68 & 1316 \\
TPU_XTAL_P & & 2.53 & 0.56 & 3.62 & 2028 \\
TPU_XTAL_F & 7800 & 1.7 & 0.28 & 2.39 & 671 \\
\hline
\end{tabular}

measured $g_{\text {local }}$ in fatigued samples is always lower than that measured in the pristine counterpart, and the drop is considerably more pronounced in TPU_XTAL (which presented the lowest values of $\lambda_{1}\left(X_{2}\right)$ measured in DIC after fatigue in all of the area around the crack tip (Figure 6)). This result is in agreement with the suggestion of Mzabi et al. that the crack propagation resistance does depend on the strain energy available within the highly strained region at the crack tip. In the case of TPU_XTAL, the ability of crystallization under strain and the corresponding remarkable increase in the modulus with applied stretch not only reduces the local strain but also the elastic energy available to propagate the crack compared to TPU_SOFT (where SIC is absent and the only strengthening mechanism at the crack tip is the fragmentation of HD). Differently from SBR and other typical vulcanized elastomers, the peculiar feature of the TPU is their ability to maintain an excellent reversible elasticity in the bulk (necessary for applications), while retaining the ability to plastically deform at high strain to reduce the severity of the strain concentration at the crack tip. A similar mechanism has been recently shown for polyampholyte hydrogels, where a straindependent structural change is also active. ${ }^{26}$

\section{CONCLUSIONS}

The cyclic fatigue behavior and structural evolution of notched samples of two commercial soft TPU with nearly identical small strain moduli but different large strain behavior have been investigated at room temperature in the pure-shear geometry $^{3}$ and reported as $\mathrm{d} c / \mathrm{d} n$ vs $G$. For both TPU, the presence of a strain gradient in an area close to the loaded crack was shown to generate a self-strengthening nonuniform spatial organization of the TPU microstructure above a threshold value of strain. The more highly strained region at the crack tip becomes stiffer than the bulk, reducing the intensity of the strain concentration and the elastic energy available at the crack tip. As a result of this weak strain concentration, the crack propagation resistance in TPU is markedly increased compared to classical vulcanized elastomers. Furthermore, the presence of strain-induced crystallization in one of the TPU improves the cyclic fatigue resistance by reinforcing the crack tip in a similar way to that in stretched natural rubbers with the main difference that the phenomenon in TPU is not completely reversible and some residual crystals are still visible in unloaded TPU. Defining a local elastic strain energy $g_{\text {local }}$ based on the methodology of Mzabi et al., we further show that a lower $g_{\text {local }}$ leads to a lower value of $\mathrm{d} c / \mathrm{d} n$ at the same applied $\lambda$.

However, when $\lambda_{\max }$ exceeds a critical value of stretch, the HD restructuration and some damage (suggested by the equatorial streak) start to occur within the whole sample and no longer selectively at the crack tip. Under those loading 
conditions, the entire material would experience a straininduced stiffening effect without the formation of a hard core protecting the crack tip leading to a higher crack propagation rate.

Our study clearly shows that this self-strengthening mechanism due to localized crack tip plasticity is active in commercial TPU and is responsible for their remarkable enhanced fatigue resistance at high strains. Interestingly, the value of such a localized stiffening mechanism may be more general and a similar localized strengthening has been observed for microphase-separated polyampholyte hydrogels. ${ }^{26}$

\section{ASSOCIATED CONTENT}

\section{SI Supporting Information}

The Supporting Information is available free of charge at https://pubs.acs.org/doi/10.1021/acs.macromol.1c00934.

Additional details on the injection process and in situ 2D $\mathrm{X}$-ray image of TPU_SOFT (PDF)

\section{AUTHOR INFORMATION}

\section{Corresponding Authors}

Matteo Ciccotti - Sciences et Ingénierie de la Matiere Molle, ESPCI Paris, Université PSL, CNRS, Sorbonne Université, 75005 Paris, France; Email: matteo.ciccotti@espci.psl.eu

Costantino Creton - Sciences et Ingénierie de la Matière Molle, ESPCI Paris, Université PSL, CNRS, Sorbonne Université, 75005 Paris, France; 이이.org/0000-00020177-9680; Email: costantino.creton@espci.psl.eu

\section{Authors}

Giorgia Scetta - Sciences et Ingénierie de la Matière Molle, ESPCI Paris, Université PSL, CNRS, Sorbonne Université, 75005 Paris, France; (1) orcid.org/0000-0002-5757-203X

Eric Euchler - Leibniz-Institut für Polymerforschung Dresden e.V., 01069 Dresden, Germany

Jianzhu Ju - Sciences et Ingénierie de la Matière Molle, ESPCI Paris, Université PSL, CNRS, Sorbonne Université, 75005 Paris, France

Nathan Selles - Laboratoire de Recherches et de Contrôle du Caoutchouc et des Plastiques, 94408 Vitry-sur-Seine, France

Patrick Heuillet - Laboratoire de Recherches et de Contrôle du Caoutchouc et des Plastiques, 94408 Vitry-sur-Seine, France

Complete contact information is available at:

https://pubs.acs.org/10.1021/acs.macromol.1c00934

\section{Notes}

The authors declare no competing financial interest.

\section{ACKNOWLEDGMENTS}

The $\mathrm{PhD}$ work of Giorgia Scetta was jointly funded by the French ANRT and the LRCCP. The authors are grateful to Dr. Matthias Schwartzkopf for the access to P03 DESY beamline and to Dr. Dario Cavallo for his support on the interpretation of X-ray data. They are indebted to Dr. Matthias Gerst, Dr. Elke Marten, and Mr. Stephan Dohmen from BASF AG for kindly providing the TPU samples. They also thank Stephane Delaunay for injecting the samples and Mohamed Hanafi for the chemical characterization. Costantino Creton has received funding from the European Research Council (ERC) under the European Union's Horizon 2020 research and innovation program under grant agreement AdG No 695351.

\section{REFERENCES}

(1) Bonart, R. X-Ray Investigations Concerning the Physical Structure of Cross-Linking in Segmented Urethane Elastomers. J. Macromol. Sci., Part B: Phys. 1968, 2, 115-138.

(2) Petrović, Z. S.; Ferguson, J. Polyurethane Elastomers. Prog. Polym. Sci. 1991, 16, 695-836.

(3) Scetta, G.; Selles, N.; Heuillet, P.; Ciccotti, M.; Creton, C. Cyclic Fatigue Failure of TPU Using a Crack Propagation Approach. Polym. Test. 2021, 97, No. 107140.

(4) Bai, R.; Yang, J.; Suo, Z. Fatigue of Hydrogels. Eur. J. Mech. A/ Solids 2019, 74, 337-370.

(5) Mzabi, S.; Berghezan, D.; Roux, S.; Hild, F.; Creton, C. A Critical Local Energy Release Rate Criterion for Fatigue Fracture of Elastomers. J. Polym. Sci. Part B Polym. Phys. 2011, 49, 1518-1524.

(6) Martinez, J. R. S.; Toussaint, E.; Balandraud, X.; Le, J.; Berghezan, D.; Martinez, J. R. S.; Toussaint, E.; Balandraud, X.; Cam, J. Le.; Heat, D. B. Heat and Strain Measurements at the Crack Tip of Filled Rubber under Cyclic Loadings Using Full-Field Techniques. In Mechanics of Materials; Elsevier, 2015; Vol. 81, pp 62-71.

(7) Yeh, F.; Hsiao, B. S.; Sauer, B. B.; Michel, S.; Siesler, H. W. InSitu Studies of Structure Development during Deformation of a Segmented Poly(Urethane-Urea) Elastomer. Macromolecules 2003, 36, 1940-1954.

(8) Rahmawati, R.; Masuda, S.; Cheng, C. H.; Nagano, C.; Nozaki, S.; Kamitani, K.; Kojio, K.; Takahara, A.; Shinohara, N.; Mita, K.; Uchida, K.; Yamasaki, S. Investigation of Deformation Behavior of Thiourethane Elastomers Using in Situ X-Ray Scattering, Diffraction, and Absorption Methods. Macromolecules 2019, 52, 6825-6833.

(9) Kojio, K.; Matsuo, K.; Motokucho, S.; Yoshinaga, K.; Shimodaira, Y.; Kimura, K. Simultaneous Small-Angle X-Ray Scattering/Wide-Angle X-Ray Diffraction Study of the Microdomain Structure of Polyurethane Elastomers during Mechanical Deformation. Polym. J. 2011, 43, 692-699.

(10) Koerner, H.; Kelley, J. J.; Vaia, R. A. Transient Microstructure of Low Hard Segment Thermoplastic Polyurethane under Uniaxial Deformation. Macromolecules 2008, 41, 4709-4716.

(11) Blundell, D. J.; Eeckhaut, G.; Fuller, W.; Mahendrasingam, A.; Martin, C. Real Time SAXS / Stress - Strain Studies of Thermoplastic Polyurethanes at Large Strains. Polymer 2002, 43, 5197-5207.

(12) Ishihara, H.; Kimura, I.; Yoshihara, N. Studies on Segmented Polyurethane-Urea Elastomers: Structure of Segmented PolyurethaneUrea Based on Poly(Tetramethylene Glycol), 4,4'-Diphenylmethane Diisocyanate, and 4,4'-Diaminodiphenylmethane. J. Macromol. Sci., Part B: Phys. 1983, 22, 713-733.

(13) Van Bogart, J. W. C.; Lilaonitkul, A.; Cooper, S. L. Morphology and Properties of Segmented Copolymers. In Multiphase Polymers; Cooper, S. L.; Gerald, M. E., Eds.; American Chemical Society, 1979; pp 3-30.

(14) Waletzko, R. S.; Korley, L. T. J.; Pate, B. D.; Thomas, E. L.; Hammond, P. T. Role of Increased Crystallinity in DeformationInduced Structure of Segmented Thermoplastic Polyurethane Elastomers with PEO and PEO-PPO-PEO Soft Segments and HDI Hard Segments. Macromolecules 2009, 42, 2041-2053.

(15) Zhu, P.; Dong, X.; Wang, D. Strain-Induced Crystallization of Segmented Copolymers: Deviation from the Classic Deformation Mechanism. Macromolecules 2017, 50, 3911-3921.

(16) Toki, S.; Hsiao, B. S.; Kohjiya, S.; Tosaka, M.; Tsou, A. H.; Datta, S. Synchrotron X-Ray Studies of Vulcanized Rubbers and Thermoplastic Elastomers. Rubber Chem. Technol. 2006, 79, 460-488.

(17) Rivlin, R. S.; Thomas, A. G. Rupture of Rubber. I. Characteristic Energy for Tearing. J. Polym. Sci. 1953, 10, 291-318.

(18) Mattia, J.; Painter, P. A Comparison of Hydrogen Bonding and Order in a Polyurethane and Poly(Urethane-Urea) and Their Blends with Poly(Ethylene Glycol). Macromolecules 2007, 40, 1546-1554.

(19) Hammersley, A. P. "FIT2D” http://www.esrf.eu/computing/ scientific/FIT2D/ (accessed March 15, 2021). 
(20) ENS. Digital image correlation which revolutionizing the domain of mechanical tests https://www.correli-stc.com/ (accessed April 15, 2021).

(21) Mzabi, S. Caractérisation et Analyse Des Mécanismes de Fracture En Fatigue Des Elastomères Chargés. PhD Thesis, 2010.

(22) Bhowmick, A. K. Threshold Fracture of Elastomers. J. Macromol. Sci., Polym. Rev. 1988, 28, 339-370.

(23) Lake, G. J.; Lindley, P. B. Mechanical Fatigue Limit for Rubber. Rubber Chem. Technol. 1966, 39, 348-364.

(24) Mars, W.; Fatemi, a. A Literature Survey on Fatigue Analysis Approaches for Rubber. Int. J. Fatigue 2002, 24, 949-961.

(25) Lake, G. J.; Lindely, P. B. Cut Growth and Fatigue of Rubbers. J. Appl. Polym. Sci. 1964, 455, 292-300.

(26) Li, X.; Cui, K.; Sun, T. L.; Meng, L.; Yu, C.; Li, L.; Creton, C.; Kurokawa, T.; Gong, J. P. Mesoscale Bicontinuous Networks in SelfHealing Hydrogels Delay Fatigue Fracture. Proc. Natl. Acad. Sci. U.S.A. 2020, 117, 7606-7612.

(27) Scetta, G.; Ju, J.; Selles, N.; Heuillet, P.; Ciccotti, M.; Creton, C. Strain Induced Strengthening of Soft Thermoplastic Polyurethanes Under Cyclic Deformation. J. Polym. Sci. 2021, 1, 685-696.

(28) Merckel, Y.; Diani, J.; Brieu, M.; Gilormini, P.; Caillard, J. Characterization of the Mullins Effect of Carbon-Black Filled Rubbers. Rubber Chem. Technol. 2011, 84, 402-414.

(29) Zhu, P.; Zhou, C.; Dong, X.; Sauer, B. B.; Lai, Y.; Wang, D. The Segmental Responses to Orientation and Relaxation of Thermoplastic Poly(Ether-Ester) Elastomer during Cyclic Deformation: An in-Situ WAXD/SAXS Study. Polymer 2020, 188, No. 122120.

(30) Statton, W. O. Microvoids in Fibers as Studied by Small-Angle Scattering of x-Rays. J. Polym. Sci. 1962, 58, 205-220.

(31) Zhang, H.; Scholz, A. K.; De Crevoisier, J.; Berghezan, D.; Narayanan, T.; Kramer, E. J.; Creton, C. Nanocavitation around a Crack Tip in a Soft Nanocomposite: A Scanning Microbeam Small Angle X-Ray Scattering Study. J. Polym. Sci., Part B: Polym. Phys. 2015, 53, 422-429.

(32) Stribeck, N.; Sapoundjieva, D.; Denchev, Z.; Apostolov, A. A.; Zachmann, H. G.; Stamm, M.; Fakirov, S. Deformation Behavior of Poly(Ether Ester) Copolymer as Revealed by Small- and Wide-Angle Scattering of X-Ray Radiation from Synchrotron. Macromolecules 1997, 30, 1329-1339.

(33) Toki, S.; Sics, I.; Hsiao, B. S.; Murakami, S.; Tosaka, M.; Poompradub, S.; Kohjiya, S.; Ikeda, Y. Structural Developments in Synthetic Rubbers during Uniaxial Deformation by In Situ Synchrotron X-Ray Diffraction. J. Polym. Sci., Part B: Polym. Phys. 2004, 42, 956-964.

(34) Demassieux, Q.; Berghezan, D.; Cantournet, S.; Proudhon, H.; Creton, C. Temperature and Aging Dependence of Strain-Induced Crystallization and Cavitation in Highly Crosslinked and Filled Natural Rubber. J. Polym. Sci., Part B: Polym. Phys. 2019, 57, 780793.

(35) Candau, N.; Stoclet, G.; Tahon, J. F.; Demongeot, A.; Yilgor, E.; Yilgor, I.; Menceloglu, Y. Z.; Oguz, O. Mechanical Reinforcement and Memory Effect of Strain-Induced Soft Segment Crystals in Thermoplastic Polyurethane-Urea Elastomers. Polymer 2021, 223, No. 123708.

(36) Kimura, I.; Ishihara, H.; Ono, H.; Yoshihara, N.; Nomura, S.; Kawai, H. Morphology and Deformation Mechanism of Segmented Poly(Urethaneureas) in Relation to Spherulitic Crystalline Textures. Macromolecules 1974, 7, 355-363. 